\title{
Tunable supercontinuum light vector vortex beam generator using a q-plate
}

\author{
Yisa S. Rumala ${ }^{1,8, *}$ Giovanni Milione, ${ }^{1,2,8}$ Thien An Nguyen, ${ }^{1,8}$ Sebastião Pratavieira, ${ }^{1,3}$ Zabir Hossain, ${ }^{1}$ \\ Daniel Nolan, ${ }^{4,8}$ Sergei Slussarenko, ${ }^{5}$ Ebrahim Karimi, ${ }^{6}$ Lorenzo Marrucci, ${ }^{5,7,8}$ and Robert R. Alfano ${ }^{1,2,8}$ \\ ${ }^{1}$ Insitute for Ultrafast Spectroscopy and Lasers (IUSL), Department of Physics, Department of Electrical Engineering, \\ City College of the City University of New York, 160 Convent Avenue, New York, New York 10031, USA \\ ${ }^{2}$ Graduate Center of the City University of New York, 365 Fifth Ave., New York, New York 10016, USA \\ ${ }^{3}$ Instituto de Física de São Carlos-Universidade de São Paulo, São Carlos, Brazil \\ ${ }^{4}$ Corning Incorporated, Sullivan Park, Corning, New York 14831, USA \\ ${ }^{5}$ Dipartimento di Fisica, Universita di Napoli “Federico II," 80126 Napoli, Italy \\ ${ }^{6}$ Department of Physics, University of Ottawa, 150 Louis Pasteur, Ottawa, Ontario K1N 6N5, Canada \\ ${ }^{7}$ CNR-SPIN, Complesso Universitario di Monte S. Angelo, 80126 Napoli, Italy \\ ${ }^{8}$ New York State Center for Complex Light, 160 Convent Ave., New York, New York 10031, USA \\ *Corresponding author: yrumala@ccny.cuny.edu
}

Received August 19, 2013; revised October 27, 2013; accepted October 27, 2013;

posted October 28, 2013 (Doc. ID 195194); published November 25, 2013

Spatially coherent multicolored optical vector vortex beams were created using a tunable liquid crystal q-plate and a supercontinuum light source. The feasibility of the q-plate as a tunable spectral filter (switch) was demonstrated, and the polarization topology of the resulting vector vortex beam was mapped. Potential applications include multiplexing for broadband high-speed optical communication, ultradense data networking, and super-resolution microscopy. (c) 2013 Optical Society of America

OCIS codes: (260.6042) Singular optics; (260.5430) Polarization; (350.2460) Filters, interference; (060.4230) Multiplexing.

http://dx.doi.org/10.1364/OL.38.005083

Optical vector vortex beams are optical modes with well defined spatial polarization modulation and topological vortex charge [1]. The q-plate [2] can naturally generate vector beams as it relies on the space-variant birefringence of transparent liquid crystals to convert light's spin angular momentum (SAM) to orbital angular momentum (OAM) $[2, \underline{3}]$. By combining a tunable q-plate [는 $\underline{6}]$ and supercontinuum light [ $\underline{7}-\underline{10}$ ], the multicolored optical vortex modes can be generated and optimized for a particular wavelength. These effects are expected to be of significance in the area of high-speed optical telecommunication, which would take advantage of the broadband frequency spectra, the beam's complex polarization topology, and the existence of controlled integer orbital angular momentum modes. An example of such an application would be a compact hybrid system that can incorporate both wavelength division multiplexing (WDM) as well as spatial mode division multiplexing in the same setup, i.e., a "hybrid mode-wavelength division multiplexer" (HMWDM) [11]. Such a system has not yet been demonstrated.

This Letter provides the first steps in the direction of an HMWDM scheme that will serve as a enabler of ultradense WDM for communication and device data networking (e.g., between computers, free space between towers, etc.). In order to execute this scheme, the following key properties will be required: (i) multicolored spatial mode in which multiple frequency channels are generated, (ii) spatially coherent optical mode, and minimized effects due to (iii) topological charge dispersion (e.g., Refs. [12,13]), (iv) spatial dispersion (e.g., Ref. [13]), or (v) chromatic dispersion (e.g., Refs. [14-16]) of the multicolored spatial mode, as well as minimized (vi) angular optical interference effects in devices used to generate the vortex (e.g., Ref. [17]).
In previous research, optical information for communication has been encoded and retrieved in frequency channels of supercontinuum light [18-21], frequency combs [22], polarization [23,24], and OAM modes of light [24-27]. Optical beams that have both a vortex spatial mode and changing polarization about the mode profile are referred to as vector beams [1], and their transformations can be conveniently described on a higher order Poincare sphere $[28,29]$. Multicolored vector beams have been created in uniaxial crystals [30,31], geometric phase elements [32], and liquid crystal droplets [33]. Nevertheless, in all these schemes, there does not exist a simple way to tune between vector vortices of different colors in the multicolored frequency spectra, which would be required for an HMWDM scheme. A tunable q-plate is a compact device that naturally generates vector vortex beams and is suitable to accomplish this task, while satisfying the requirements mentioned earlier.

A tunable multicolored light source emitting spatially coherent vector beam based on a tunable q-plate is discussed here with the goal of realizing a compact HMWDM. The output light from the q-plate consists of a superposition of optical vortex spatial modes with different colors. The space-variant polarization of the multicolored optical vortex mode was mapped point by point using Stokes polarimetry. The tunability of the q-plate using an applied electric field in conjunction with a single mode optical (SMO) fiber was used to demonstrate a tunable optical spectral filter/switch as a first step to realizing the HMWDM scheme. The q-plate is known to generate vector beams at monochromatic wavelengths $[\underline{2}, \underline{4}-\underline{6}, \underline{26}, \underline{34}]$, but to our knowledge, the properties of a coherent multicolored light source have not been studied after propagating through a q-plate. By adjusting the 
electric field across the q-plate, the transmitted optical beam profile smoothly changes its wavelength and mode structure.

The proposed HMWDM scheme involves using the polarization modulation within the vortex spatial mode to encode and retrieve information in multicolored frequency spectra. The multicolored optical wave containing frequency channels was sent through a device (i.e., q-plate) used to generate a polarization dependent modulation and vortex mode. The different states of light polarization (e.g., horizontal, vertical, diagonal) were mapped into discrete spatial points on the multicolored optical vortex mode. The vortex mode can be used to turn on and off select frequency channels. Frequency spectral channels are generated from a supercontinuum light source [18] (i.e., photonic crystal fiber [35]), whereas the q-plate selects particular frequency (wavelength) channels and spatial mode channels. This proposed multiplexing scheme can be used to "switch" between different frequency and spatial mode channels in an optical network.

The polarization topology of the coherent multicolored supercontinuum light vector vortex beam was mapped using Stokes polarimetry. A photonic crystal (PC) fiber was used to generate the supercontinuum (SC) light from which multicolored coherent light was obtained. A pulsed laser beam with a center wavelength of $800 \mathrm{~nm}$, measured pulse duration of about $130 \mathrm{fs}$, repetition rate of $76 \mathrm{MHz}$, and average power (peak power) of $310 \mathrm{~mW}$ $(31 \mathrm{~kW})$, was sent into the PC fiber. A diffraction grating was placed after the PC fiber and a slit was placed afterward to collect a single color from the first diffraction order, or all the colors from the zeroth diffraction order. The diffraction order was coupled into a SMO fiber to obtain a clean $\mathrm{TEM}_{00}$ Gaussian optical mode. This emerging light goes through a linear polarizer, then a q-plate of $q=1$ to generate a vector vortex with no net OAM, i.e., there is both $\ell=2$ and $\ell=-2$ to give a net OAM of zero. Afterward, the light goes through a linear polarizer to measure the beam's Stokes parameters. The resulting vector vortex was imaged onto a charge-coupled device (CCD) camera. The experimental setup is presented in Fig. 1(a). The electric field through the q-plate is tuned by changing the input voltage until all the vortices in the spectra are degenerate and form a single vortex of multiple colors, i.e., there is an approximate dark vortex core in the middle of the beam as seen in Fig. 2(d).

The Stokes parameter maps of the optical beam profile were obtained using a standard method described in Ref. [34]. That is, two linear polarizers were used to measure the Stokes parameters, and 4 measurements were taken with the polarizer after the q-plate oriented at $0^{\circ}, 45^{\circ}, 90^{\circ}$, and $135^{\circ}$ with respect to the polarizer before the q-plate. Stokes parameters were defined as: $S_{0}=I_{0}+I_{90}, S_{1}=I_{0}-I_{90}$, and $S_{2}=I_{45}-I_{135}$. From the measured Stokes parameters [36], the polarization at each point on a single color vector vortex mode was mapped; the results are presented in Figs. 2(a)-2(c). The Stokes parameters were measured for the following single colors: red $(617 \pm 2 \mathrm{~nm})$, yellow $(585 \pm 1 \mathrm{~nm})$, and green $(540 \pm 3 \mathrm{~nm})$. The same Stokes polarimetry was done for the multicolored vector vortex generated from the zeroth diffraction order of the diffraction

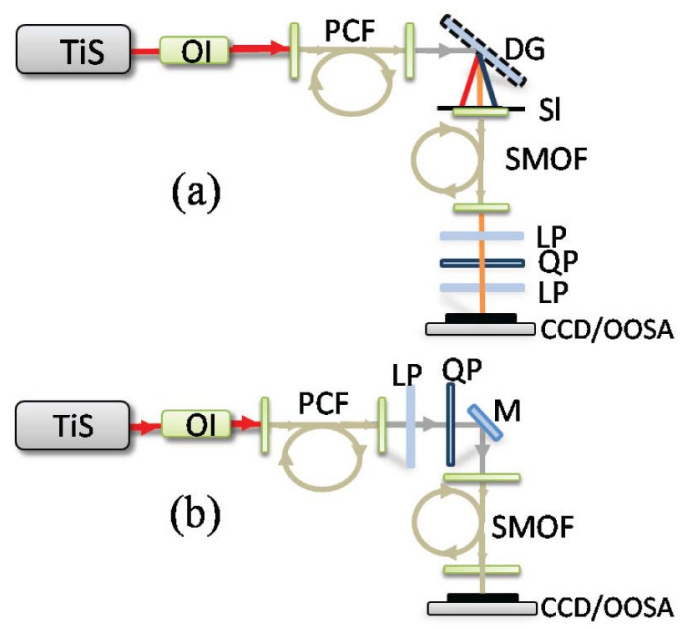

Fig. 1. Experimental setup for (a) mapping vector vortex polarization topology and (b) implementing the optical spectral filter/switch. TiS, titanium-sapphire laser; OI, optical isolator; PCF, photonic crystal fiber; DG, diffraction grating; Sl, slit; SMOF, single mode optical fiber; LP, linear polarizer; QP, q-plate; CCD, charge coupled device camera; OOSA, ocean optics spectrum analyzer; M, mirror.

grating. The results are shown in Fig. 2(d), with the corresponding wavelength spectra presented in Fig. 2(e). The spectral profile is preserved as the beam goes through the SMO fiber.

The feasibility of the q-plate as a tunable optical filter (switch) is now discussed. The experimental setup is as follows: multicolored supercontinuum light is generated using a PC fiber and made to be linearly polarized as described in the first scheme. The beam is then sent through a q-plate of $q=1$ and the light coupled into a SMO fiber, with a cutoff wavelength at $416 \mathrm{~nm}$. An objective lens with a numerical aperture of $\mathrm{NA}=0.4$ is used to couple light through free space into the SMO fiber with $\mathrm{NA}=0.12$. The resulting beam is imaged onto a CCD camera. The experimental setup is presented in Fig. 1(b).

As the external voltage applied to the q-plate is tuned from 2.50 to $2.00 \mathrm{~V}$ in steps of $0.25 \mathrm{~V}$, the q-plate is "resonant" for particular wavelengths and detuned from other wavelengths. The wavelengths that are "resonant" with the q-plate implies that all beam power will be in the vortex mode (i.e., a higher order mode), while the wavelengths that are detuned (not "resonant") from

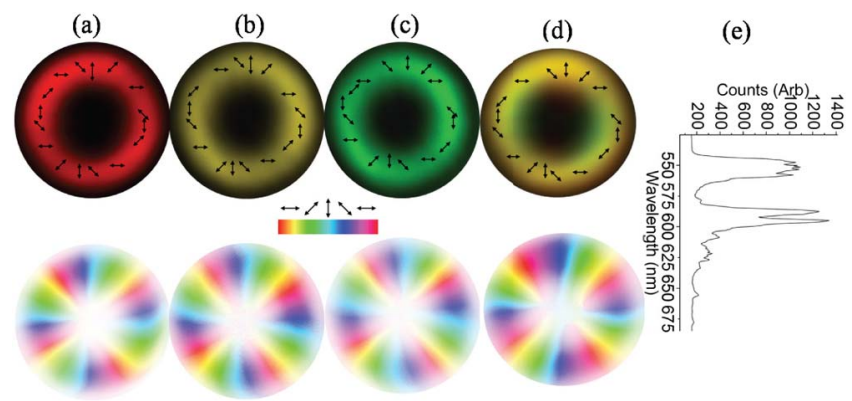

Fig. 2. Experimental intensity (upper) and polarization map (lower) of vector vortex: (a) $617 \pm 2 \mathrm{~nm}$ (red), (b) $585 \pm 1 \mathrm{~nm}$ (yellow), (c) $540 \pm 3 \mathrm{~nm}$ (green), which were extracted from (d) the multicolored vortex. (e) Spectra of multicolored vortex-(d) showing wavelength (frequency) spectra from the PC fiber. 

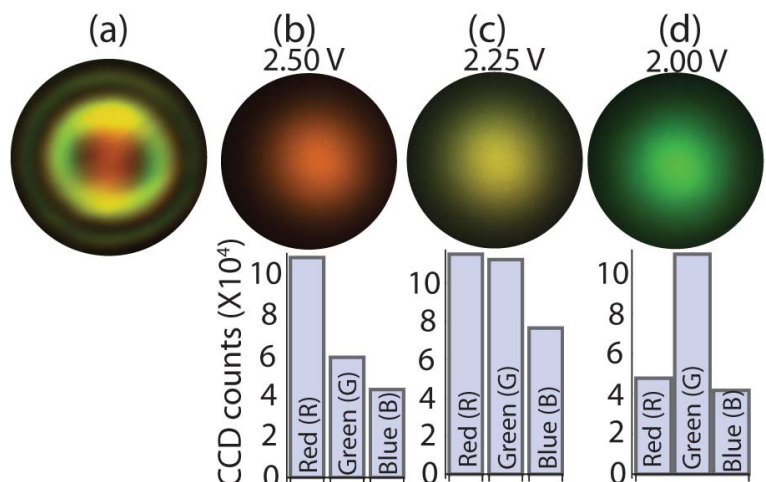

Fig. 3. Experimental data demonstrating the optical spectral filter. (a) Example of multicolored optical mode generated by the q-plate and going into the SMO fiber. As the voltage through the q-plate is tuned from 2.50 to $2.00 \mathrm{~V}$, the colors emerging from the SMO fiber change from orange to yellow to green [35] as shown in (b)-(d). Barchart of CCD counts represent the total counts for each RGB color on the CCD screen, where counts in each pixel were normalized to a value between 0 and 1, and actual CCD counts ranged from 0 to 255 .

the q-plate implies a fraction of power in the beam will be in the lowest order mode (i.e., $\mathrm{TEM}_{00}$ Gaussian mode). At different q-plate voltages, the coupling efficiency of light going into the SMO fiber will change since the SMO fiber supports only $\mathrm{TEM}_{00}$ Gaussian modes for operating wavelengths. As a result, the light coming out of the SMO fiber changes color from orange to yellow to green in incremental steps for respective voltages of 2.50, 2.25, and $2.00 \mathrm{~V}$, as presented in Figs. 3(b)-3(d). The SMO fiber is effectively acting as a discriminator for the various color channels in multicolored mode, and could be incorporated in the transmission or receiving end in the HMWDM scheme. The purity of colors in the spatially coherent optical intensity profile coming out of the SMO fiber is quantified in a barchart containing an RGB (Red, Green, Blue) color analysis [37]; and also presented in Figs. 3(b)-3(d). This demonstrates the feasibility of the device as a tunable spectral filter (or optical switch) using the SAM to OAM conversion of light by the q-plate in conjunction with a SMO fiber. The scheme can therefore serve as an optical switch to tune between different frequency channels in the proposed HMWDM scheme described earlier, and it should be possible to implement in free space.

In conclusion, optical vector vortex beams have been created using a multicolored supercontinuum laser light source and an electrically tunable q-plate. Together with a single mode fiber, this effect can be used as a wavelength selective optical filter, or "optical switch," to move between different frequency channels in a communication network, especially in the proposed HMWDM scheme. The scheme is not limited to optical communications, but can be applied in the development of cost effective stimulated emission depletion (STED) fluorescence microscopy systems [38-40] with sub-diffraction resolution, such that tunning is achieved between different colors of the optical vortices by simply changing the electric field going through the q-plate.

The work is supported in part by ARO and Corning, Inc. S. S. and L. M. acknowledge support from the Future
Emerging Technologies program of the European Commission, Grant No. 255914, PHORBITECH.

\section{References and Notes}

1. Q. Zhan, Adv. Opt. Photon. 1, 1 (2009).

2. L. Marrucci, C. Manzo, and D. Paparo, Phys. Rev. Lett. 96, 163905 (2006).

3. G. Biener, A. Niv, V. Kleiner, and E. Hasman, Opt. Lett. 27, 1875 (2002).

4. E. Karimi, B. Piccirillo, E. Nagali, L. Marrucci, and E. Santamato, Appl. Phys. Lett. 94, 231124 (2009).

5. B. Piccirillo, V. D'Ambrosio, S. Slussarenko, L. Marrucci, and E. Santamato, Appl. Phys. Lett. 97, 241104 (2010).

6. S. Slussarenko, A. Murauski, T. Du, V. Chigrinov, L. Marrucci, and E. Santamato, Opt. Express 19, 4085 (2011).

7. R. R. Alfano and S. L. Shapiro, Phys. Rev. Lett. 24, 592 (1970).

8. R. R. Alfano and S. L. Shapiro, Phys. Rev. Lett. 24, 584 (1970).

9. R. R. Alfano and S. L. Shapiro, Phys. Rev. Lett. 24, 1217 (1970).

10. R. R. Alfano, The Supercontinuum Laser Source, 2nd ed. (Springer, 2006)

11. Y. S. Rumala, S. Pratavieira, G. Milione, T. A. Nguyen, Z. Hossain, D. Nolan, E. Karimi, S. Slussarenko, L. Marrucci, and R. R. Alfano, in Proceedings of Frontiers in Optics (Optical Society of America, 2013), paper FTu1F.

12. G. A. Swartzlander, Jr. and J. Schmit, Phys. Rev. Lett. 93, 093901 (2004).

13. Y. Tokizane, K. Oka, and R. Morita, Opt. Express 17, 14517 (2009).

14. J. Leach and M. J. Padgett, New J. Phys. 5, 154 (2003).

15. M. E. Anderson, H. Bigman, L. E. E. de Araujo, and J. L. Chaloupka, J. Opt. Soc. Am. B 29, 1968 (2012).

16. H. I. Sztul, V. Kartazayev, and R. R. Alfano, Opt. Lett. 31, 2725 (2006).

17. Y. S. Rumala and A. E. Leanhardt, J. Opt. Soc. Am. B 30, 615 (2013).

18. I. Zeylikovich, V. Kartazaev, and R. R. Alfano, J. Opt. Soc. Am. B 22, 1453 (2005).

19. H. Takara, Opt. Photon. News 13(2), 48 (2002).

20. O. Boyraz and M. N. Islam, J. Lightwave Technol. 20, 1493 (2002).

21. H. Takara, T. Ohara, T. Yamamoto, H. Masuda, M. Abe, H. Takahashi, and T. Morioka, Electron. Lett. 41, 270 (2005).

22. P. J. Delfyett, S. Gee, M.-T. Choi, H. Izadpanah, W. Lee, S. Ozharar, F. Quinlan, and T. Yilmaz, J. Lightwave Technol. 24, 2701 (2006).

23. S. Bhandare, D. Sandel, B. Milivojevic, A. Hidayat, A. A. Fauzi, H. Zhang, S. K. Ibrahim, F. Wust, and R. Noe, IEEE Photon. Technol. Lett. 17, 914 (2005).

24. J. Wang, J. Y. Yang, I. M. Fazal, N. Ahmed, Y. Yan, H. Huang, Y. Ren, Y. Yue, S. Dolinar, M. Tur, and A. E. Willner, Nat. Photonics 6, 488 (2012).

25. N. Bozinovic, Y. Yue, Y. Ren, M. Tur, P. Kristensen, H. Huang, A. E. Willner, and S. Ramachandran, Science 340, 1545 (2013).

26. E. Karimi, L. Marrucci, C. de Lisio, and E. Santamato, Opt. Lett. 37, 127 (2012).

27. G. Gibson, J. Courtial, M. J. Padgett, M. Vasnetsov, V. Pas'Ko, S. M. Barnett, and S. Franke-Arnold, Opt. Express 12, 5448 (2004).

28. G. Milione, H. I. Sztul, D. A. Nolan, and R. R. Alfano, Phys. Rev. Lett. 107, 053601 (2011).

29. G. Milione, S. Evans, D. A. Nolan, and R. R. Alfano, Phys. Rev. Lett. 108, 190401 (2012). 
30. T. A. Fadeyeva, V. G. Shvedov, Y. V. Izdebskaya, A. V. Volyar, E. Brasselet, D. N. Neshev, A. S. Desyatnikov, W. Krolikowski, and Y. S. Kivshar, Opt. Express 18, 10848 (2010).

31. Y. A. Egorov, T. A. Fadeyeva, and A. V. Volyar, J. Opt. A 6, S217 (2004).

32. A. Niv, G. Biener, V. Kleiner, and E. Hasman, Opt. Lett. 32, 847 (2007).

33. E. Brasslet, N. Murazawa, H. Misawa, and S. Juodkazis, Phys. Rev. Lett. 103, 103903 (2009).

34. F. Cardano, E. Karimi, S. Slussarenko, L. Marrucci, C. Lisio, and E. Santamato, Appl. Opt. 51, C1 (2012).

35. J. M. Dudley, G. Genty, and S. Coen, Rev. Mod. Phys. 78, 1135 (2006).

36. Measurement of $S_{3}$ is not necessary as there appears to be greater than $90 \%$ agreement between $S_{0}^{2}$ and $S_{1}^{2}+S_{2}^{2}$ for pixels with a large number of counts (i.e., $S_{0}^{2} \approx S_{1}^{2}+S_{2}^{2}$ ). Therefore, polarization of the vector vortex mode is expected to be mostly linear.
37. The purity of colors is quantified in the barchart below each image. The barchart is a measure of the response of the CCD to the relative red (R), blue (B), and green (G) colors present in the beam. All other visible colors are extrapolated from these RGB colors. Hence, the barcharts in Figs. 3(b) and 3(d) have the most counts in red and green, respectively, and show clear discrimination from other colors in the beam. Since yellow is largely a mixture of red and green counts in pixels, these two colors will have the most counts compared to other colors in the beam, which is consistent with the CCD sensor spectral response to different colors. The CCD data sheets can be found at http://ww2 .ptgrey.com/USB2/chameleon.

38. K. I. Willig, S. O. Rizzoli, V. Westphal, R. Jahn, and S. W. Hell, Nature 440, 935 (2006).

39. D. Wildanger, E. Rittweger, L. Kastrup, and S. W. Hell, Opt. Express 16, 9614 (2008).

40. T. J. Gould, E. B. Kromann, D. Burke, M. J. Booth, and J. Bewersdorf, Opt. Lett. 38, 1860 (2013). 